\title{
La Facultad de Ciencias Pecuarias y Agroindustriales de la Universidad Politécnica Salesiana
}

P. Miguel Ulloa

sta Facultad es, sin duda, una preciosa rama de la frondosa higuera, denominada Politécnica Salesiana. La UPS tiene hondas raíces porque la semilla técnica fue sembrada en 1888.

En 1927 aún estaba vigente la ley prohibitiva de instalar en el Ecuador Centros Educativos Católicos. Por esta razón, "entre celos y recelos", el Padre Carlos Crespi abrió en Cuenca, Yanuncay, la Escuela Particular Agronómica Salesiana, en la Quinta María Auxiliadora. Puso al frente de ella el Experto Agrónomo Padre Antonio Gardini, el cual tuvo la colaboración de los expertos en Agricultura, Coadjutores Salesianos Sebastián Turello, José Zublena y José Vicini.

La finalidad de esta Escuela Privada era formar Coadjutores Salesianos, expertos en Agricultura, para enseñar a los shuar a cultivar correctamente en las fértiles tierras amazónicas.

El 30 de Enero de 1950 fue reconocida como "Escuela Complementaria Agrícola", por el Ministerio Economía. En 1958 pasó a la dependencia del Ministerio de Fomento.

EI Instituto Técnico Cornelio Merchán de Cuenca, en 1956 fue ascendido a la categoría de Colegio Técnico. Desgraciadamente el 19 de Julio un voraz incendio consumió el edificio y los talleres del Merchán. Dios permitió dar un paso adelante con la construcción y nuevo equipamiento del nuevo Técnico frente al Aeropuerto.

Sobre estas sólidas bases fue creada la Universidad Politécnica Salesiana (UPS), el 5 de Agosto de 1994, con sede en Cuenca, mediante el Decreto-Ley N${ }^{\circ} 65$ del Congreso Nacional. En el mismo Decreto, Art 3 dice: La Universidad Politécnica Salesiana tendrá las siguientes especializaciones. C) Facultad de Ciencias Pecuarias y Agroindustriales, con las Escuelas de Tecnología, Zootecnia y de Ingeniería Industrial.

A consecuencia de la reestructuración de los Ministerios del Estado, el 16 de Febrero de 1959, el Colegio Agronómico Salesiano fue asignado al Ministerio de Educación.

El historiador P. Guerriero dice: "El plantel está considerado, en su género, entre los mejores del país, por estudios, dotación y organización. Pero, para su mayor desarrollo convenía sacarlo de Cuenca. El trasladarlo a Uzhupud fue un verdadero acierto.

Después del aluvión de La Josefina y de su heroica reconstrucción, la Comunidad de Uzhupud se dijo: ¿Por qué no podemos pertenecer a la Unidad Educativa de la UPS, transformando nuestro plantel en Instituto Superior Agronómico Salesiano? Así fue y así lo hicimos con el Acuerdo Ministerial 4026 del 29 de Agosto de 1997

También fue otro acierto porque, siendo autónoma la Universidad Politécnica Salesiana, fácilmente pudo crear sobre esta base la Facultad de Ciencias Pecuarias y Agroindustriales. El gestor principal y primer Decano fue el Lcdo. Padre Fausto Jara.

El lunes primero de Octubre 1997 iniciamos el año lectivo con 72 alumnos matriculados: 60 varones y 12 señoritas, distribuidos en dos paralelos $A$ y $B$.

La crónica nos recuerda el Personal Directivo y Docente: Lcdo. Padre Fausto Jara, Decano; Ing. Agro. Marcelo Jimbo, Subdecano; Directores de Producción, Salesianos Peritos Agrónomos Señores Luis Gannis y Juan Lunardi; Director de Investigación Botánica, Padre Angel Andretta, y Secretario de la Facultad, Lcdo. Josué Benigno Ortiz.

Entre los 13 profesores se encuentran el Padre Virgilio Barbessi, Director de la Comunidad, y el Padre Miguel Ulloa, Vicario Superior, encargados de la Formación Moral y Religiosa los primeros Profesores Cofundadores: Ing. Freddy Portilla, Lic. Ramiro Tapia, Lic. Reinaldo Tapia, Ing. Manuel Morales, Ing. Daniel Ortiz, Ing. Carlos Vanegas, Dr. Luis Ayala, TIgo. Leonidas Espinoza, Ing. Hernán Avilés, Ing. Marcelo Jimbo e Ing. Narcisa Larriva.

La solemne Inauguración se llevó a cabo e 31 de Octubre en los Salones de la Hostería Uzhupud, con la asistencia del Rector de la UPS Padre Luciano Bellini y del Consejo Directivo de la UPS, de los señores Alcaldes de los Cantones del Oriente Azuayo, de la Comunidad Salesiana y del Profesorado de la Facultad y del Instituto, de los alumnos de la Facultad y de sus padres de familia.

Por motivos de salud y de estudios, en 1999 el Padre Fausto Jara fue enviado a Roma por lo Superiores. Ocuparon su lugar, como Decano el Ing. Freddy Portilla Farfán y como Rector del I.T.S.A.S. el Lcdo. Oswaldo Pulla.

La única Comunidad Salesiana de Paute tiene dos obras: la de Uzhupud donde funcionan el Instituto y la Facultad, y la de Yugmacay donde se brinda atención a los jóvenes del Hogar Juvenil Don Bosco. Por carecer de espacio físico para las clases, en el 2000 hemos creído conveniente pasar la sección universitaria a Yugmacay. Desde luego, los universitarios realizan sus prácticas en Uzhupud.

Para la enseñanza práctica, la Entidad cuenta con 225 hectáreas en Uzhupud, más 40 hectáreas de terreno en Yugmacay; presenta, igualmente, un buen lote de semovientes: 70 cabezas de ganado holstein, gran cantidad de cerdos de raza fina, de llamas, chivos, ovejas, cuyes, conejos, aves de corral, abejas, peces, ranas, caracoles, lombrices y viveros para la reforestación de la zona.

Los alumnos, desde el cuarto curso en adelante, se entrenan en los laboratorios y en el manejo de la maquinaria agrícola.

En el presente Año Lectivo, la Facultad cuenta con 217 alumnos en los cinco cursos. El primero de Febrero pasado se incorporaron los primeros 23 tecnólogos. Próximamente tendremos los primeros Ingenieros Agropecuarios Industriales.

La semilla sembrada por la Virgen Auxiliadora ha germinado hasta hacerse un gigantesco árbol que hoy cubre todo el País. Sus múltiples ramas producen óptimos frutos de buenos cristianos y honrados ciudadanos, preparados, sobre todo, en áreas técnicas y agrícolas. 